\title{
Erratum to: Irradiation with visible light enhances the antibacterial toxicity of silver nanoparticles produced by laser ablation
}

\author{
Matthew Ratti $^{1}$ - J. J. Naddeo ${ }^{1}$ - Yuying Tan ${ }^{1} \cdot$ Julianne C. Griepenburg $^{1}$ • \\ John Tomko ${ }^{1}$ Cory Trout $^{1} \cdot$ Sean M. O'Malley ${ }^{1,2} \cdot$ Daniel M. Bubb $^{1,2}$. \\ Eric A. Klein ${ }^{2,3}$
}

Published online: 31 March 2016

(C) Springer-Verlag Berlin Heidelberg 2016

Erratum to: Appl. Phys. A (2016) 122:346

DOI 10.1007/s00339-016-9935-8

The original version of this article unfortunately contained a mistake.

There were 2 wrong references in the text. The correct references are given here.

1. Bioavailability assays were performed using E. coli strain MC1061 (pSLcueR/pDNPcopAlux), a kind gift from Anne Kahru (National Institute of Chemical Physics and Biophysics, Estonia) [14].

2. The sulfur-containing organic ligand, dithizone (Sigma), forms a complex with $\mathrm{Ag}^{+}$ions with an absorption peak at roughly $465 \mathrm{~nm}$ [11].

The original article was corrected.

The online version of the original article can be found under doi:10.1007/s00339-016-9935-8.

\footnotetext{
Eric A. Klein

eric.a.klein@rutgers.edu

1 Physics Department, Rutgers University-Camden, Camden, NJ 08102, USA

2 Center for Computational and Integrative Biology, Rutgers University-Camden, Camden, NJ 08102, USA

3 Biology Department, Rutgers University-Camden, Camden, NJ 08102, USA
} 